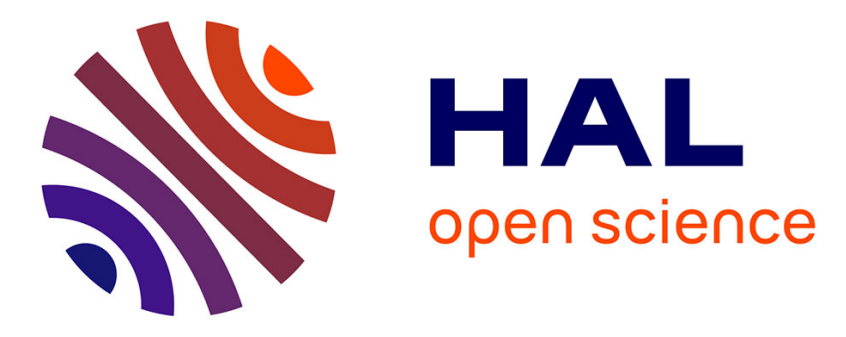

\title{
Directional surface enhanced Raman scattering on gold nano-gratings
}

\author{
Raymond Gillibert, Mitradeep Sarkar, Jean-François Bryche, Ryohei \\ Yasukuni, Julien Moreau, Mondher Besbes, Grégory Barbillon, Bernard \\ Bartenlian, Michael Canva, Marc Lamy de La Chapelle
}

\section{To cite this version:}

Raymond Gillibert, Mitradeep Sarkar, Jean-François Bryche, Ryohei Yasukuni, Julien Moreau, et al.. Directional surface enhanced Raman scattering on gold nano-gratings. Nanotechnology, 2016, 27 (11), pp.115202. 10.1088/0957-4484/27/11/115202 . hal-01534295

\section{HAL Id: hal-01534295 \\ https://hal-iogs.archives-ouvertes.fr/hal-01534295}

Submitted on 9 Oct 2017

HAL is a multi-disciplinary open access archive for the deposit and dissemination of scientific research documents, whether they are published or not. The documents may come from teaching and research institutions in France or abroad, or from public or private research centers.
L'archive ouverte pluridisciplinaire HAL, est destinée au dépôt et à la diffusion de documents scientifiques de niveau recherche, publiés ou non, émanant des établissements d'enseignement et de recherche français ou étrangers, des laboratoires publics ou privés. 


\title{
Directional Surface Enhanced Raman Scattering on gold nano-gratings
}

\author{
Raymond Gillibert ${ }^{1,2}$, Mitradeep Sarkar ${ }^{3}$, Jean-François \\ Bryche $^{4,3}$, Ryohei Yasukuni ${ }^{1}$, Julien Moreau ${ }^{3}$, Mondher \\ Besbes $^{3}$, Grégory Barbillon ${ }^{4}$, Bernard Bartenlian ${ }^{4}$, Michael \\ Canva $^{3,5}$, Marc Lamy de la Chapelle ${ }^{1}$ \\ ${ }^{1}$ Université Paris 13, Sorbonne Paris Cité, Laboratoire CSPBAT, CNRS, (UMR 7244), \\ 74 rue Marcel Cachin, F-93017 Bobigny, France \\ ${ }^{2}$ HORIBA Jobin Yvon S.A.S. Villeneuve d'Ascq, 231 rue de Lille 59650 Lille, France \\ ${ }^{3}$ Institut d'Optique Graduate School, Laboratoire Charles Fabry CNRS UMR 8501, \\ 91227 Palaiseau, France \\ ${ }^{4}$ Université Paris-Sud, Institut d'Électronique Fondamentale CNRS UMR 8622, \\ Bâtiment 220, 91405 Orsay Cedex, France \\ ${ }^{5}$ Université de Sherbrooke, Laboratoire Nanotechnologie Nanosystème, LN2, UMI \\ CNRS 3463, 3IT, Canada \\ E-mail: marc.lamydelachapelle@univ-paris13.fr
}

\begin{abstract}
Directional plasmon excitation and SERS emission were demonstrated for 1D and 2D gold nanostructure arrays deposited on flat gold layer. Extinction spectrum of both arrays exhibits intense resonance bands that are red shifted when the incident angle is increased. Systematic extinction analysis of different grating periods revealed that this band can be assigned to a propagated surface plasmon of the flat gold surface that fulfills the Bragg condition of the arrays (Bragg mode). Directional SERS measurements demonstrated that the SERS intensity can be improved by one order of magnitude, when the Bragg mode positions matched with either the excitation or the Raman wavelengths. Hybridized numerical calculations of Finite Element Method and Fourier Modal Method also proved the presence of Bragg mode plasmon and illustrated that the enhanced electric field of the Bragg mode is particularly localized on the nanostructures regardless of their size.
\end{abstract}

Keywords: SERS, Plasmon, grating, gold. 


\section{Introduction}

Metallic nanostructures have proved to be very useful for various applications owing to their capability to confine electromagnetic (EM) fields at the nanometric scales by the excitation of surface plasmon resonances (SPR) $[1,2,3,4]$. One of the most relevant applications of such effect is the surface enhanced Raman scattering (SERS), which is a highly sensitive spectroscopic technique for the detection of molecules and the analysis of their chemical structures $[5,6,7,8,9,10,11]$. In fact, the SERS effect has been observed for a large variety of metallic nanostructures $[12,13,14,15,16,17]$, fabricated by various techniques such as chemical synthesis, electrochemical etching and nanolithography[18, 19, 20, 21, 22, 23, 24, 25, 26].

Recently, directionality of SERS in regularly-structured or flat metal substrates has been reported in several publications as a useful technique to control SERS efficiency. First, basic angular dependence of the SERS signal was reported for flat metallic surface in Kretschmann configuration. In such works, it was shown that the maximum of SERS intensity is reached when the angle matches the angle of the SPR excitation of the flat metal surface $[27,28,29]$. In this case, the SERS signal dependence is only due to the angular dependence of the plasmon excitation. For nanostructured surfaces, some advanced angular plasmonic and SERS studies have been performed. For instance, Chu, Y. et al. have studied a double resonance SERS substrate,[30] namely a combination of a gold nanodisks array and a gold film with a thin dielectric spacer. This SERS substrate shows that local field enhancement around the nanodisk is very sensitive to the excitation angle. This effect is essentially due to a coupling of the localized surface plasmon (LSP) of the gold nanodisks with the propagative surface plasmon (PSP) of the gold film. Moreover, Baumberg et al. have reported a comprehensive extinction and SERS angular study on two-dimensional nanoholes array in a gold film [31]. A directionality of the SERS emission was found and explained by the excitation of plasmonic modes originating from the periodicity of the nanoholes. Thus, these previous studies featured SERS directionality as a result of directional excitations of complex surface plasmon modes. However, their general behaviors have not been clearly determined yet because of the lack of systematic studies of their structural parameters. In addition, little were pointed out for the coupling of SERS emission processes with such plasmon modes.

In this paper, we present a complete study of the angular dependence of the plasmon excitation and of the SERS signal. This study has been performed with 1D and 2D gold nanostructures arrays deposited on flat gold film, which sustain both LSP and PSP. In order to examine complex surface plasmon modes excited in the fabricated SERS substrates and their contribution to the SERS intensities, the grating period of the gold nanostructure array as well as the excitation wavelength have been systematically changed. The nanostructured arrays were realized by electron beam lithography that allows the fabrication of regular and reproducible nanostructures leading to predictable EM field enhancement[32, 33, 34, 35, 36, 37, 38, 39, 40, 41]. Numerical simulations were carried out to calculate the EM field intensity and its near-field distribution, and were 

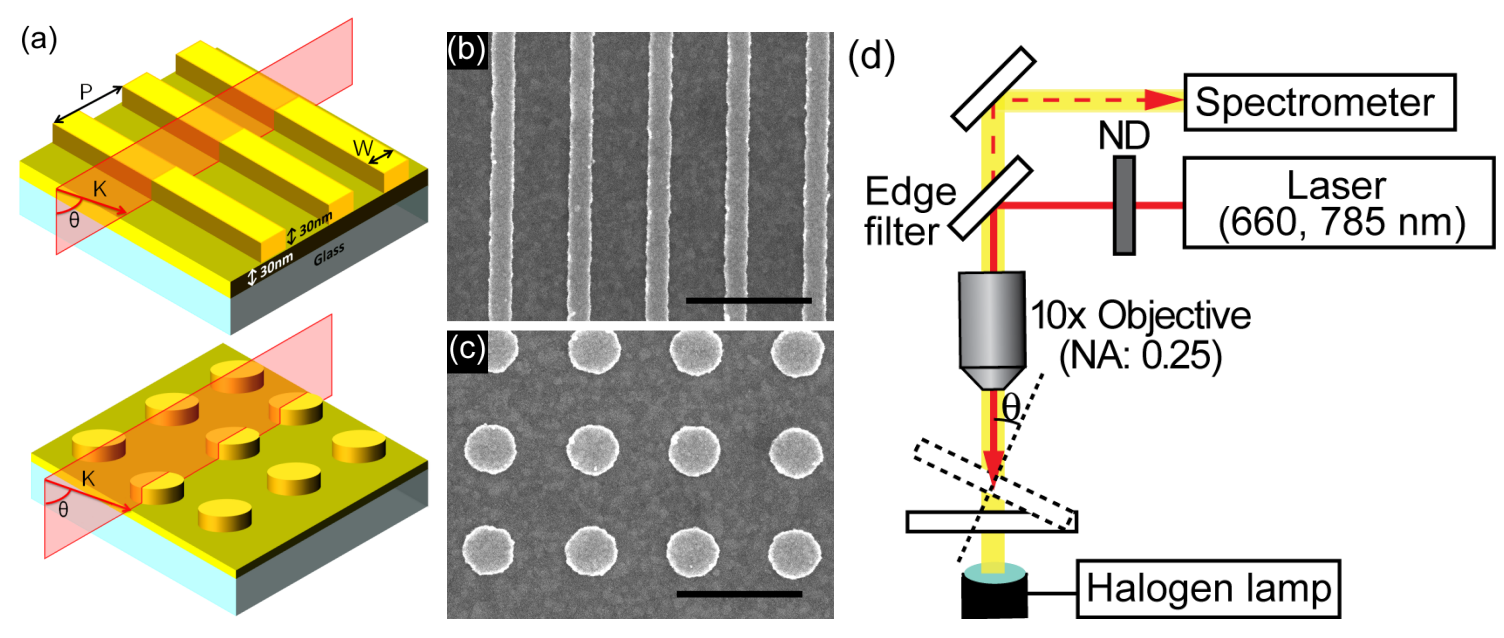

Figure 1. (a) Schematic of the nanolines and nanodisks substrates. (b and c) SEM images of nanolines (b) and nanodisks (c) with a period of $400 \mathrm{~nm}$. The scale bars are $500 \mathrm{~nm}$ long. (d) Schematics of the SERS and extinction configuration with respect to the substrate. For SERS, both excitation and collection are done with a $10 \times$ microscope objective $(\mathrm{NA}=0.25)$. For extinction measurement, the excitation is done in transmission configuration using white light illumination from below the sample whereas the transmitted light is collected with the $10 \times$ objective. For all experiments, the excitation polarization is transverse magnetic (TM) and the wave-vector $\mathrm{K}$ is parallel to the grating axis, meaning an azimuthal angle of $0^{\circ}$.

compared with our experimental results. In this paper, we discuss the directional SERS as an intrinsic property of all periodic structures, which can be simply explained by the excitation of PSP which satisfy the Bragg condition of the arrays at certain angle of incidences. This work demonstrates that an intensity gain of one order of magnitude can be obtained just by tilting the sample and that the collection angle must be optimized to reach the highest SERS signals.

\section{Results and discussion}

\subsection{Directional extinction analysis}

As shown on the figures $1 \mathrm{a}, \mathrm{b}$ and $\mathrm{c}$, we used two different nanostructures for these studies: nanolines (30 $\mathrm{nm}$ in height and $100 \mathrm{~nm}$ in width) and nanodisks (30 $\mathrm{nm}$ in height and with a diameter of $220 \mathrm{~nm}$ ) with an underlying thin gold film (30 nm in height). The width or the diameter of the nanostructures is fixed for all experiments. Directional extinction spectra were recorded using a low numerical aperture objective $(\mathrm{NA}=0.25)$ and by tilting the sample with respect to the optical axis as shown in Figure 1c.

As shown on Figure 2a, the extinction spectra of nanoline array exhibit two resonance peaks: one between 650 and $800 \mathrm{~nm}$ and the other beyond $850 \mathrm{~nm}$. The positions of both peaks are highly dependent on the excitation angle and on the period of the nanolines arrays (Figure 2b). The resonance position of both peaks undergoes a linear redshift with the angle of incidence. One can also notice that the slope of the curves increase 

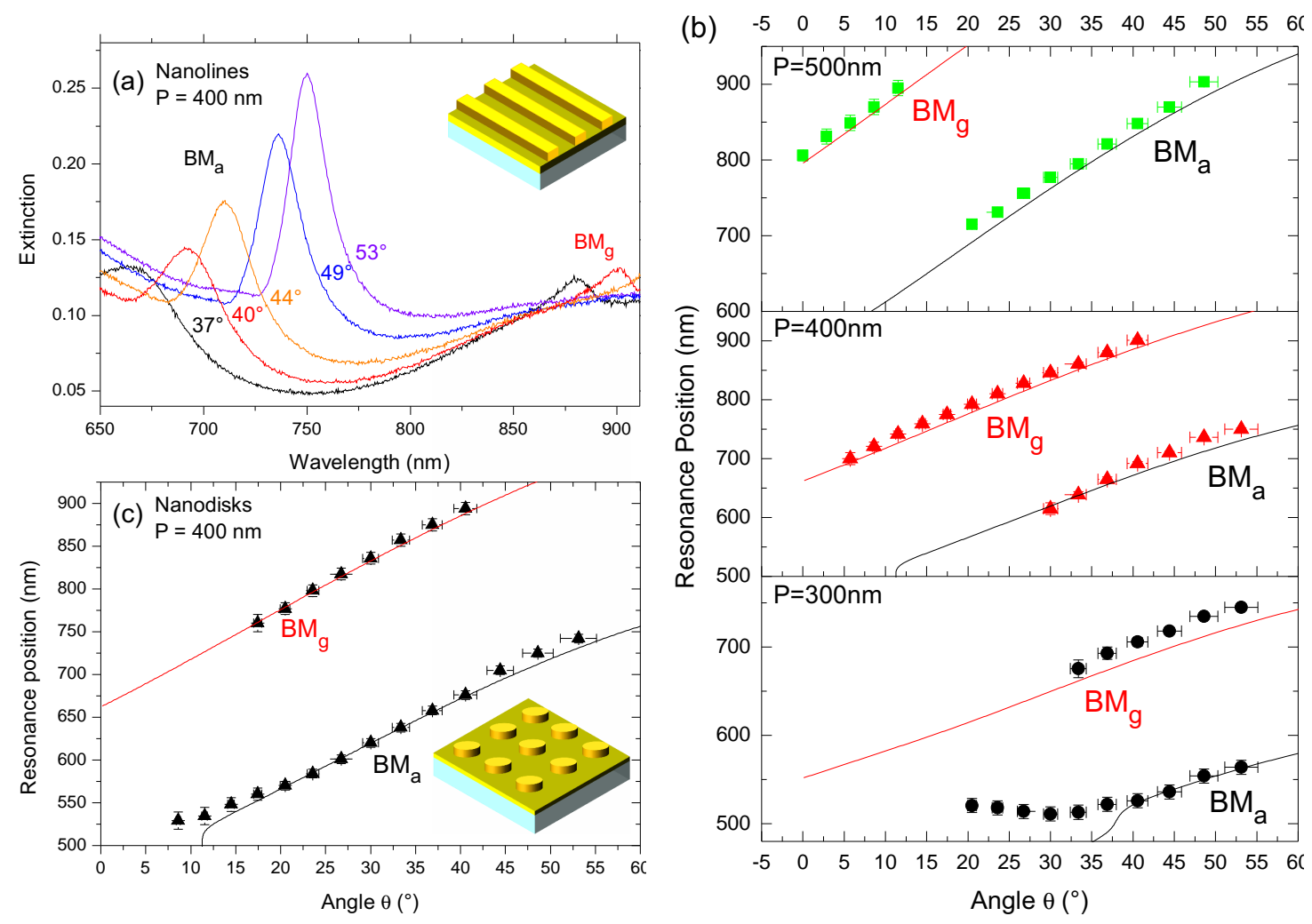

Figure 2. (a) Extinction spectra for different $\theta$ angles on nanolines with a $400 \mathrm{~nm}$ grating period. (b) Plasmon resonance position as a function of $\theta$ angle for three different periods for nanolines and (c) for nanodisks with a $400 \mathrm{~nm}$ grating period. The solids lines represent the analytical solution of equation 2 for the corresponding grating periods.

when the array period increases from $300 \mathrm{~nm}$ to $500 \mathrm{~nm}$. We can then observe a clear dependence of the resonance with the measurement angle and the array period. Similar behavior is also observed for the 2D array with nanodisks: linear dependence of the resonance position with the measurement angle. In this case, resonance positions of both the peaks are close to those measured for the 1D array of nanolines. Thus, it indicates that the resonance position is independent of the shape of the nanostructure, but is only related to the period of the array.

Similar phenomenon has been reported by $\mathrm{Xu}$ et al. on 1D metal grating structures [42]. They explained the shift of resonance position as an excitation of different modes of the PSP. In our case, the excitation of the two plasmonic modes could be explained as follows. Because of the presence of the flat gold film below the grating[43, 44], PSP can be excited. Its wave-vector in the plane parallel to the metal-dielectric interface $\left(k_{x}\right)$ can be written as: [45]

$$
k_{s p p}=k_{0} \sqrt{\frac{n_{d}^{2} \varepsilon_{m}(\omega)}{n_{d}^{2}+\varepsilon_{m}(\omega)}}
$$

where $\varepsilon_{m}(\omega)$ is the complex permittivity of the metal, $k_{0}=2 \pi / \lambda$ is the free space 
wave-vector for the wavelength $\lambda$ and $n_{d}$ is the refractive index of the dielectric-medium. $n_{d}$ is equal to $n_{a}$ (refractive index of air), when the PSP is excited at the metal-air interface and is equal to $n_{g}$ (refractive index of the glass substrate) when the PSP is excited at the metal-glass interface.

When the wave-vector of the PSP matches the Bragg vector $\left(k_{B}=2 \pi / P\right.$, where $\mathrm{P}$ is the grating constant) of the array, a highly confined electromagnetic field is created around the nanostructures due to the constructive interference of the PSP at the positions of the nanostructures. This mode is called the Bragg mode (BM) in analogy to Bragg diffraction in periodic structures. The condition for the excitation of the BM can be written as [46]:

$$
k_{B M}=k_{s p p} \pm k_{B}
$$

where $k_{B M}$ is the in-plane wave-vector given by $k_{0} \sin \theta$. The analytical solution of equation 2 (shown as solid lines on Figures $2 \mathrm{~b}$ and $2 \mathrm{c}$ ) is in good agreement, for all periods and geometries, with the experimental position of the plasmon resonances. This confirms that the resonance only depends on the array period and on the measurement angle and not on the nanostructure shape. The mode at lower wavelengths $\left(B M_{a}\right)$ is due to the fulfillment by the PSP of the condition at the metal-air interface, and the mode at higher wavelengths $\left(B M_{g}\right)$ is due to the fulfillment of the condition at the metal-glass interface. Bragg modes of higher order are not observed since the grating periods are smaller than the wavelengths of our measured spectral range. It should be noted that the LSP of both nanolines and nanodisks are visible around $550 \mathrm{~nm}$ and their position exhibit no angular dependence (data not shown).

\subsection{Directional SERS intensity Analysis}

To measure the SERS signal, thiophenol was used as a probe molecule because of its high Raman cross-section and the strong affinity of the thiol group towards the gold surface. Such molecule also creates homogenous self-assembled monolayer giving a reproducible SERS signal [47]. Several intense Raman peaks of thiophenol are observable at 419, 1000, 1024, 1075 and $1575 \mathrm{~cm}^{-1}$. The most intense band is observable at $1075 \mathrm{~cm}^{-1}$ and was used to measure the SERS intensity. Figure 3 shows the SERS spectra of thiophenol recorded on nanolines with a grating period of $400 \mathrm{~nm}$ for two excitation angles, $\theta=0^{\circ}$ and $34^{\circ}$ (Excitation wavelength $=660 \mathrm{~nm}$ ). A clear increase of the SERS signal is noticed for the excitation angle of $34^{\circ}$ indicating an angular dependence of the SERS intensity.

To demonstrate this, the SERS intensity was experimentally recorded as a function of the incident angle $\theta$ in the range $\left[0,50^{\circ}\right]$ at three different wavelengths $(633,660$ and $785 \mathrm{~nm}$ ) on the nanolines array for three different grating periods (300, 400, $500 \mathrm{~nm})$, and on the nanodisks array for a grating period of $400 \mathrm{~nm}$ (Figure 4).

Strong variations of the SERS intensity were observed depending on the angle with intensity peaks at specific angles. At these angles, the intensity is between five and 


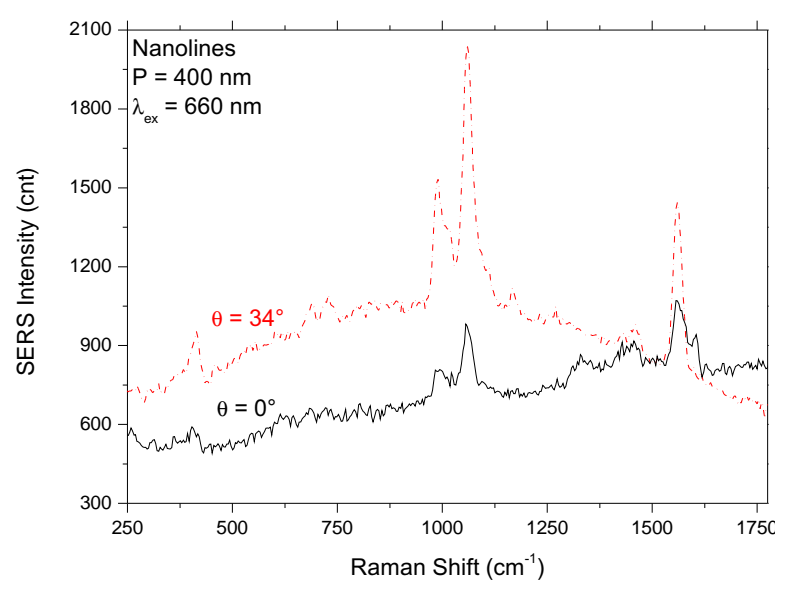

Figure 3. SERS spectra of thiophenol recorded on the nanolines (width $=100 \mathrm{~nm}$ and grating period $=400 \mathrm{~nm}$ ) at $660 \mathrm{~nm}$. Excitation angles $\theta$ are $0^{\circ}$ (solid black line) and $34^{\circ}$ (dashed red line).

ten times higher than the one measured at normal incidence $\left(\theta=0^{\circ}\right)$. The systematic study reveals that large intensity peaks for the SERS signal are obtained when the $B M_{a}$ are excited whereas smaller peaks for the SERS signal is observed, when the $B M_{g}$ are excited. Hence, it can be considered that the improvement of SERS intensity at a certain incidence angle is caused by the excitation of the $B M_{a}$. The angle of incidence which satisfies the condition for excitation of the BM increases, when the excitation wavelength is redshifted or when the grating period is increased.

The smaller values of the SERS intensity at the positions of $B M_{g}$ are due to the confinement of the EM near-field of the $B M_{g}$ at the metal/glass interface. Furthermore its decay length is not long enough to produce a high field enhancement at the interface between gold and air, where the thiophenol molecules are located. We have also observed that the extinction values corresponding to the $B M_{g}$ were lower than those for the $B M_{a}$.

It is also interesting to notice that we observe a second maximum at the angle, where the emission wavelength $\left(\lambda_{R}\right)$ matches the BM resonance conditions marked by vertical dashed lines. This indicates that the Raman scattering couples with the BMs. Thus the Raman Scattering depends on the directional emission of BMs.

Finally, we found similar behaviors between 1D nanolines (Figures 4 d,e,f) and 2D nanodisks (Figures $4 \mathrm{j}, \mathrm{k}, \mathrm{i}$ ), when they have the same $400 \mathrm{~nm}$ grating period even though they have different geometries. Those angular dependencies of the SERS intensity imply that they would be sensitive to only the grating period, but not to the geometry of the nanostructure.

In order to explain the experimental results, the angular dependence of the SERS intensity was numerically simulated based on the local EM field intensity calculation using the hybrid method of the Finite Element Method (FEM) and the Fourier Modal Method (FMM)[48, 49]. The SERS intensity is generally considered to be proportional to the local EM field intensities at the excitation wavelength $\left(\lambda_{e x}\right)$ and the Raman 

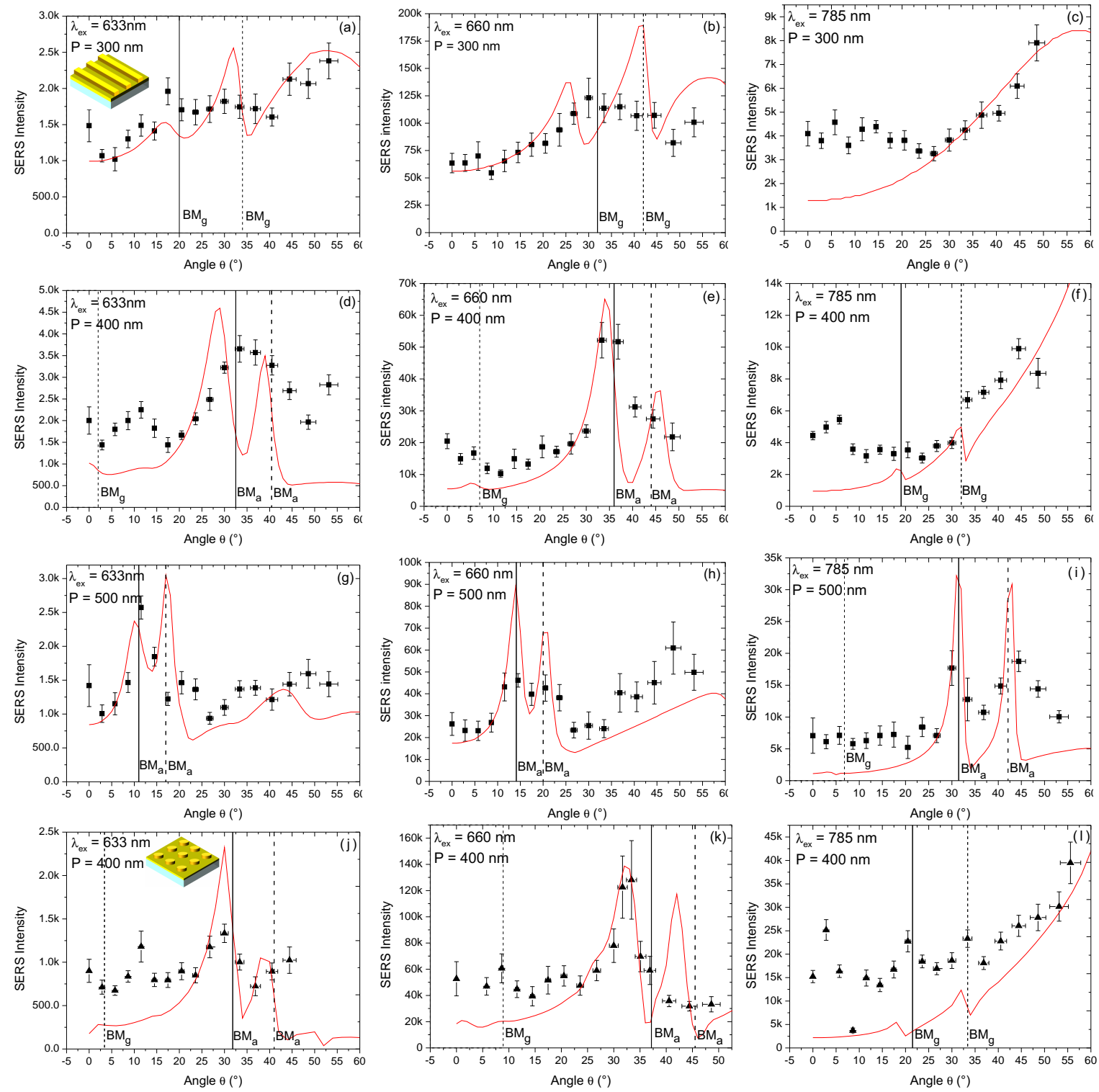

Figure 4. Experimental (dots) and numerical (solid red line) angular dependences of the SERS intensities at different excitation wavelengths $(633,660$ and $785 \mathrm{~nm}$ ) on nanolines (a-i) with grating periods from $300 \mathrm{up}$ to $500 \mathrm{~nm}$ and on nanodisks $(\mathrm{j}-\mathrm{l})$ with a grating period of $400 \mathrm{~nm}$. Vertical lines indicate the angles of BM excitation at the excitation (solid line) and emission (dashed) wavelengths.

scattering wavelength $\left(\lambda_{R}\right)$. By introducing the excitation angle $\theta_{e x}$ and the Raman collection angle $\theta_{R}$, the SERS intensity $I_{S E R S}$ in our experiments can be analytically expressed as [51]:

$$
I_{S E R S} \propto E_{e x}^{2}\left(\lambda_{e x}, \theta_{e x}\right) \times E_{R}^{2}\left(\lambda_{R}, \theta_{R}\right)
$$

where $E_{e x}^{2}$ and $E_{R}^{2}$ are the local EM field enhancement normalized by the EM field intensity of incident light $E_{0}^{2}$ at the excitation wavelength $\left(\lambda_{e x}\right)$ and Raman emission wavelength $\left(\lambda_{R}\right)$ respectively $[50,51] . \theta_{0}$ and $\theta_{R}$ are same angles in our case. The right side of equation 3 represents the SERS gain in the EM enhancement mechanism. 

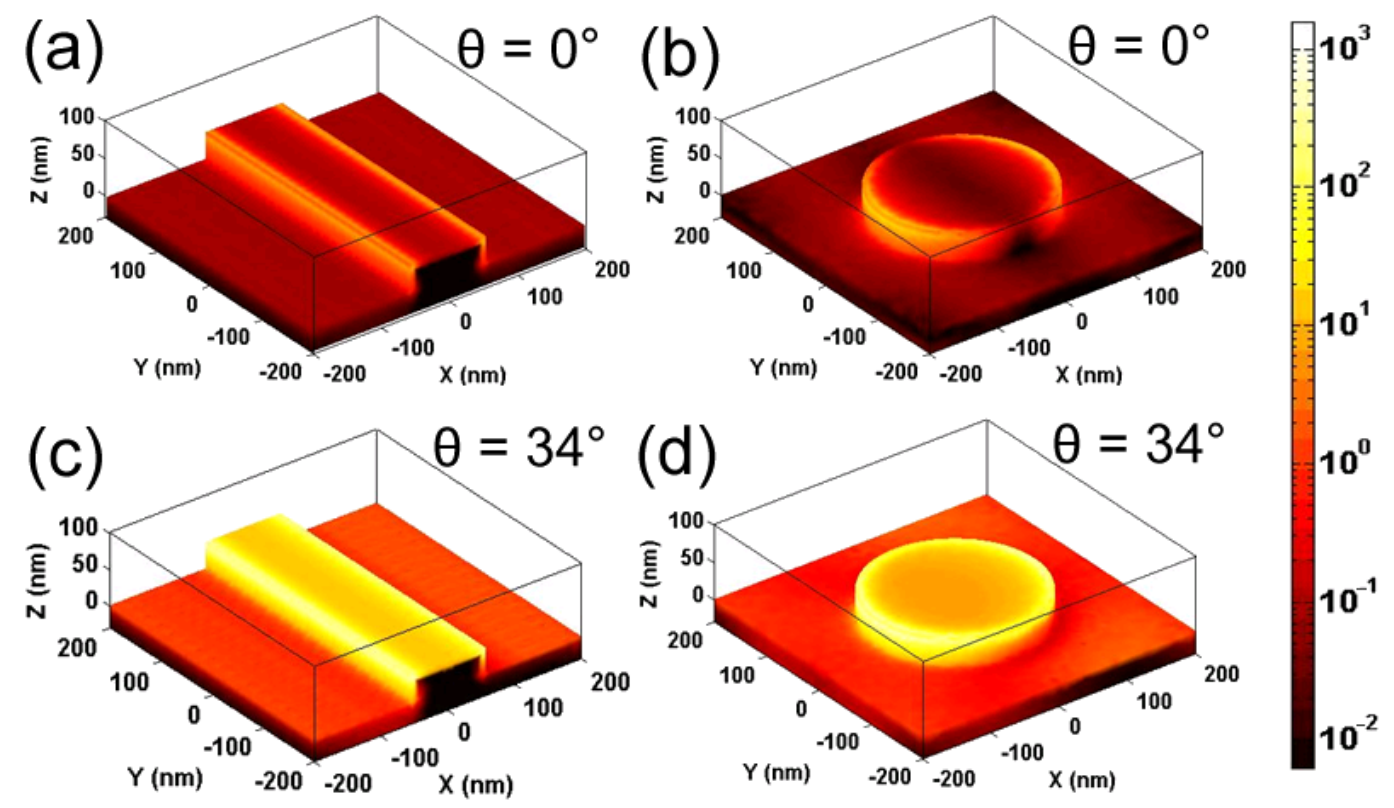

Figure 5. Spatial distributions of the calculated SERS gain for $100 \mathrm{~nm}$ width nanolines and for $220 \mathrm{~nm}$ diameter nanodisks at $660 \mathrm{~nm}$ excitation. Periodicity is set as $400 \mathrm{~nm}$. The incidence and emission angles $\theta$ were $0^{\circ}(\mathrm{a}, \mathrm{b})$ and $34^{\circ}(\mathrm{c}, \mathrm{d})$. The excitation polarization is transverse magnetic (TM) and the wave-vector $\mathrm{K}$ is parallel to the grating axis.

In Figure 5, spatial distributions of the calculated SERS gain at the gold-air interface, where the molecules are adsorbed, are illustrated for the $100 \mathrm{~nm}$ width nanolines and the $220 \mathrm{~nm}$ diameter nanodisks considering a grating period of $400 \mathrm{~nm}$. For the calculation, the excitation wavelength at $660 \mathrm{~nm}$ was used, and the incident angles were taken at $0^{\circ}$ and $34^{\circ}$ to the plane of normal incidence. The $34^{\circ}$ angle corresponds to the maximum SERS intensity for these specific experimental conditions (Figures $4 \mathrm{e}$ and $\mathrm{k}$ ). When $\theta_{e x}$ and $\theta_{R}$ are $0^{\circ}$, the effective SERS gain is only observable at the edge of nanostructures, which can be assigned to a small contribution of LSP excitation. In contrast, when $\theta_{e x}$ and $\theta_{R}$ are $34^{\circ}$, a SERS gain stronger by one order of magnitude is observed compared to the $0^{\circ}$ angle. Moreover, the SERS gain comes from the entire nanostructures and is not limited to the borders. In addition, the SERS signal is also enhanced on the gold film between the cylinders. Although the signal from the nanostructures would dominate the SERS intensity, the contribution from the flat surface might not be ignored. This means that nearly all the molecules deposited on the gold surface contribute to the SERS signal due to the excitation of the BM.

The experimentally obtained angular dependency of SERS intensity was calculated from the integral of the SERS gain over the whole surface shown in Figure 5. The total SERS gain is as follows:

$$
G_{\text {total }}=\int_{S_{\text {struct }}} E_{\text {ex }}^{2}\left(\lambda_{e x}, \theta_{e x}\right) \times E_{R}^{2}\left(\lambda_{R}, \theta_{R}\right) \mathrm{d} S
$$


The calculated $G_{\text {total }}$ is superimposed in Figure 4 (red solid lines). The numerical calculations reproduces the experimental peak positions and the angular dependency in each conditions. Therefore, the coupling of the excitation and emission lights with the BM is the main factor that improves the SERS signal in our substrate. However, for the experimental peaks, the intensity contrast is much lower and the full widths at half maximum of the peaks are larger than the ones observed with the simulated peaks. This could be explained by the fact that we used a microscope objective with a non-zero NA, which means that the excitation and the collection angles have a certain distribution. Thus, the decrease of the contrast and the broadening of the peaks result from the lower angular resolution. In addition, the roughness of the nanostructure is not taken into account in the simulation, and can also induces a higher SERS signal at unexpected angles, decreasing the experimental intensity contrast. However, these simulations confirm the high directivity of the SERS signal for specific and precise angles.

\section{Conclusion}

We demonstrated that in the case of a nanostructure grating over a metallic film, the propagating surface plasmon couples with grating, which results in the Bragg mode. Due to this coupling, there is a specific resonance angle for each wavelength of interacting light that induces a strong increase of the SERS signal. This induces a directivity of the SERS emission and thus might be useful for future applications in SERS. Thus, in a setup where one needs to work at long distances, the knowledge of this behavior might be critical for the optimization of the SERS signal.

\section{Methods}

Thin gold layer of $30 \mathrm{~nm}$ was deposited on a glass substrate by electron beam evaporation. Then gold nanostructure arrays were designed on the gold film by electron beam lithography following by a lift-off process. Surface functionalization was done by immersing the substrate for $150 \mathrm{~min}$ into a solution of thiophenol $\left(10^{-4} \mathrm{M}\right)$ dissolved in ethanol. SERS spectra were recorded with commercial confocal Raman microspectrometers with a set of continuous wavelength lasers $(\lambda=633,660$ and $785 \mathrm{~nm})$. A Horiba Scientific LabRam spectrometer was used at $633 \mathrm{~nm}$ and a Horiba Scientific Xplora spectrometer was used at 660 and $785 \mathrm{~nm}$. For each spectrometers, the Raman measurements were carried out in backscattering configuration through a $10 \times$ objective $(\mathrm{NA}=0.25)$. Incident laser power was measured at the sample position and adjusted to 3 $\mathrm{mW}$ with a neutral density filter (ND) for all laser wavelengths. Extinction spectra were recorded with the same spectrometer as SERS measurements, after removing the edge filters. A halogen lamp was used as white light source in transmission configuration (figure 1). A reference spectrum was acquired on the flat gold surface for each incident angle far from the nanostructures and the extinction was calculated as the logarithm of the ratio between reference spectrum and the spectrum acquired on the grating log $\left(I_{0} / I\right)$. 


\section{Acknowledgement}

The authors acknowledge ANR P2N (ANR-12-NANO-0016) and the support of the French Government for partial funding of the project in which this work takes place and ANRT Association Nationale de la Recherche et de la Technologie for partial funding. Also the authors thanks l'Initiative d'Excellence de Paris Saclay for funding.

[1] William L Barnes, Alain Dereux, and Thomas W Ebbesen. Surface plasmon subwavelength optics. Nature, 424(6950):824-830, 2003.

[2] Jaysen Nelayah, Mathieu Kociak, Odile Stéphan, F Javier García de Abajo, Marcel Tencé, Luc Henrard, Dario Taverna, Isabel Pastoriza-Santos, Luis M Liz-Marzán, and Christian Colliex. Mapping surface plasmons on a single metallic nanoparticle. Nat. Phys., 3(5):348-353, 2007.

[3] TJ Davis, DE Gómez, and KC Vernon. Simple model for the hybridization of surface plasmon resonances in metallic nanoparticles. Nano lett., 10(7):2618-2625, 2010.

[4] Yue Bing Zheng, Brian Kiraly, Sarawut Cheunkar, Tony Jun Huang, and Paul S Weiss. Incidentangle-modulated molecular plasmonic switches: a case of weak exciton-plasmon coupling. Nano Lett., 11(5):2061-2065, 2011.

[5] Gary Braun, Seung Joon Lee, Mark Dante, Thuc-Quyen Nguyen, Martin Moskovits, and Norbert Reich. Surface-enhanced Raman spectroscopy for DNA detection by nanoparticle assembly onto smooth metal films. J. Am. Chem. Soc., 129(20):6378-6379, 2007.

[6] Catalina David, Nicolas Guillot, Hong Shen, Timothée Toury, and Marc Lamy de la Chapelle. Sers detection of biomolecules using lithographed nanoparticles towards a reproducible sers biosensor. Nanotechnology, 21(47):475501, 2010.

[7] J Bernard Heyns, L Malia Sears, Robert C Corcoran, and Keith T Carron. Sers study of the interaction of alkali metal ions with a thiol-derivatized dibenzo-18-crown-6. Anal. Chem., 66(9):1572-1574, 1994.

[8] Nicolas Guillot and Marc Lamy de la Chapelle. Lithographied nanostructures as nanosensors. J. Nanophotonics, 6(1):064506-1, 2012.

[9] M. Cottat, N Lidgi-Guigui, F. Hamouda, B. Bartenlian, D. Venkataraman, R. S. Marks, T. W. J. Steele, and M. Lamy de la Chapelle. Highly sensitive detection of paclitaxel by surface enhanced raman scattering. J. Opt., 17(11):114019, 2015.

[10] Maximilien Cottat, Cristiano DAndrea, Ryohei Yasukuni, Natalia Malashikhina, Ruta Grinyte, Nathalie Lidgi-Guigui, Barbara Fazio, Angela Sutton, Olivier Oudar, Nathalie Charnaux, et al. High sensitivity, high selectivity sers detection of mnsod using optical nanoantennas functionalized with aptamers. J. Phys. Chem. C, 119(27):15532-15540, 2015.

[11] Souhir Boujday, Marc Lamy de la Chapelle, Johannes Srajer, and Wolfgang Knoll. Enhanced vibrational spectroscopies as tools for small molecule biosensing. Sensors, 15(9):21239-21264, 2015.

[12] Marc Lamy de la Chapelle, Nicolas Guillot, Benoît Frémaux, Hong Shen, and Timothée Toury. Novel apolar plasmonic nanostructures with extended optical tunability for sensing applications. Plasmonics, 8(2):475-480, 2013.

[13] Olivier Péron, Emmanuel Rinnert, T Toury, Marc Lamy de la Chapelle, and Chantal Compere. Quantitative sers sensors for environmental analysis of naphthalene. Analyst, 136(5):1018-1022, 2011.

[14] Chad E Talley, Joseph B Jackson, Chris Oubre, Nathaniel K Grady, Christopher W Hollars, Stephen M Lane, Thomas R Huser, Peter Nordlander, and Naomi J Halas. Surface-enhanced Raman scattering from individual au nanoparticles and nanoparticle dimer substrates. Nano Lett., 5(8):1569-1574, 2005.

[15] Maximilien Cottat, Nathalie Lidgi-Guigui, Inga Tijunelyte, Grégory Barbillon, Frédéric Hamouda, Philippe Gogol, Abdelhanin Aassime, Jean-Michel Lourtioz, Bernard Bartenlian, and Marc Lamy 
de la Chapelle. Soft uv nanoimprint lithography-designed highly sensitive substrates for sers detection. Nanoscale Res. Lett., 9(623):623, 2014.

[16] Jian Ye, Fangfang Wen, Heidar Sobhani, J Britt Lassiter, Pol Van Dorpe, Peter Nordlander, and Naomi J Halas. Plasmonic nanoclusters: near field properties of the fano resonance interrogated with sers. Nano lett., 12(3):1660-1667, 2012.

[17] Jianping Xie, Qingbo Zhang, Jim Yang Lee, and Daniel IC Wang. The synthesis of sers-active gold nanoflower tags for in vivo applications. ACS Nano, 2(12):2473-2480, 2008.

[18] DJ White, AP Mazzolini, and PR Stoddart. Fabrication of a range of sers substrates on nanostructured multicore optical fibres. J. Raman Spectrosc., 38(4):377-382, 2007.

[19] Daniel M Kuncicky, Brian G Prevo, and Orlin D Velev. Controlled assembly of sers substrates templated by colloidal crystal films. J. Mater. Chem., 16(13):1207-1211, 2006.

[20] Daniel R Ward, Nathaniel K Grady, Carly S Levin, Naomi J Halas, Yanpeng Wu, Peter Nordlander, and Douglas Natelson. Electromigrated nanoscale gaps for surface-enhanced Raman spectroscopy. Nano lett., 7(5):1396-1400, 2007.

[21] Hui Wang, Carly S Levin, and Naomi J Halas. Nanosphere arrays with controlled sub-10-nm gaps as surface-enhanced Raman spectroscopy substrates. J. Am. Chem. Soc., 127(43):14992-14993, 2005.

[22] Cristiano DAndrea, Jorg Bochterle, Andrea Toma, Christian Huck, Frank Neubrech, Elena Messina, Barbara Fazio, Onofrio M Marago, Enzo Di Fabrizio, Marc Lamy de La Chapelle, et al. Optical nanoantennas for multiband surface-enhanced infrared and raman spectroscopy. ACS Nano, 7(4):3522-3531, 2013.

[23] Donald R Herriott and George R Brewer. Electron-beam lithography machines. Electron-Beam Technology in Microelectronic Fabrication, pages 141-216, 1980.

[24] Johan Grand, Sergei Kostcheev, J-L Bijeon, Marc Lamy de la Chapelle, P-M Adam, Anna Rumyantseva, Gilles Lérondel, and Pascal Royer. Optimization of sers-active substrates for near-field raman spectroscopy. Synth. Met., 139(3):621-624, 2003.

[25] Hong Shen, Nicolas Guillot, Jérémy Rouxel, Marc Lamy de la Chapelle, and Timothée Toury. Optimized plasmonic nanostructures for improved sensing activities. Opt. Express, 20(19):2127821290, 2012.

[26] Jean-François Bryche, Raymond Gillibert, Grégory Barbillon, Mitradeep Sarkar, Anne-Lise Coutrot, Frédéric Hamouda, Abdelhanin Aassime, Julien Moreau, Marc Lamy de la Chapelle, Bernard Bartenlian, and Canva Michael. Density effect of gold nanodisks on the sers intensity for a highly sensitive detection of chemical molecules. J. Mater. Sci, 50:6601-6607, 2015.

[27] Haibo Li, Shuping Xu, Yu Liu, Yuejiao Gu, and Weiqing Xu. Directional emission of surfaceenhanced Raman scattering based on a planar-film plasmonic antenna. Thin Solid Films, 520(18):6001-6006, 2012.

[28] Stefan A Meyer, Baptiste Auguié, Eric C Le Ru, and Pablo G Etchegoin. Combined SPR and SERS microscopy in the Kretschmann configuration. J. Phys. Chem. A, 116(3):1000-1007, 2012.

[29] Dominique Barchiesi, Thomas Grosges, Florent Colas, and Marc Lamy de la Chapelle. Combined SPR and SERS: Otto and Kretschman configurations. J. Opt., 17(11):114009, 2015.

[30] Yizhuo Chu, Wenqi Zhu, Dongxing Wang, and Kenneth B. Crozie. Beamed Raman: directional excitation and emission enhancement in a plasmonic crystal double resonance SERS substrate. Opt. Express, 19(21):20054-20068, 2011.

[31] Jeremy J Baumberg, Timothy A Kelf, Yoshihiro Sugawara, Suzanne Cintra, Mamdouh E Abdelsalam, Phillip N Bartlett, and Andrea E Russell. Angle-resolved surface-enhanced Raman scattering on metallic nanostructured plasmonic crystals. Nano lett., 5(11):2262-2267, 2005.

[32] M Kahl, E Voges, S Kostrewa, C Viets, and W Hill. Periodically structured metallic substrates for sers. Sens. Actuators, B, 51(1):285-291, 1998.

[33] N Félidj, J Aubard, G Lévi, JR Krenn, M Salerno, G Schider, B Lamprecht, A Leitner, and FR Aussenegg. Controlling the optical response of regular arrays of gold particles for surfaceenhanced raman scattering. Phys. Rev. B, 65(7):075419, 2002. 
[34] N Felidj, J Aubard, G Levi, JR Krenn, A Hohenau, G Schider, A Leitner, and FR Aussenegg. Optimized surface-enhanced raman scattering on gold nanoparticle arrays. Appl. Phys. Lett., 82(18):3095-3097, 2003.

[35] N Guillot, H Shen, B Frémaux, Olivier Péron, Emmanuel Rinnert, T Toury, and Marc Lamy de la Chapelle. Surface enhanced Raman scattering optimization of gold nanocylinder arrays: Influence of the localized surface plasmon resonance and excitation wavelength. Appl. Phys. Lett., 97(2):023113, 2010.

[36] Nicolas Guillot and Marc Lamy de la Chapelle. The electromagnetic effect in surface enhanced raman scattering: Enhancement optimization using precisely controlled nanostructures. J. Quant. Spectrosc. Radiat. Transfer, 113(18):2321-2333, 2012.

[37] Johan Grand, M Lamy de La Chapelle, J-L Bijeon, P-M Adam, Alexandre Vial, and Pascal Royer. Role of localized surface plasmons in surface-enhanced raman scattering of shape-controlled metallic particles in regular arrays. Phys. Rev. B, 72(3):033407, 2005.

[38] Laurent Billot, M Lamy de La Chapelle, A-S Grimault, Alexandre Vial, Dominique Barchiesi, J-L Bijeon, P-M Adam, and Pascal Royer. Surface enhanced raman scattering on gold nanowire arrays: evidence of strong multipolar surface plasmon resonance enhancement. Chem. Phys. Lett., 422(4):303-307, 2006.

[39] Sameh Kessentini, Dominique Barchiesi, Cristiano DAndrea, Andrea Toma, Nicolas Guillot, Enzo Di Fabrizio, Barbara Fazio, Onofrio M Marago, Pietro G Gucciardi, and Marc Lamy de la Chapelle. Gold dimer nanoantenna with slanted gap for tunable lspr and improved sers. $J$. Phys. Chem. C, 118(6):3209-3219, 2014.

[40] A-S Grimault, Alexandre Vial, and M Lamy De La Chapelle. Modeling of regular gold nanostructures arrays for sers applications using a 3d fdtd method. Appl. Phys. B, 84(12):111-115, 2006.

[41] A-S Grimault, Alexandre Vial, Johan Grand, and M Lamy de la Chapelle. Modelling of the nearfield of metallic nanoparticle gratings: localized surface plasmon resonance and sers applications. J. Microsc., 229(3):428-432, 2008.

[42] Bin-Bin Xu, Zhuo-Chen Ma, Huan Wang, Xue-Qing Liu, Yong-Lai Zhang, Xu-Lin Zhang, Ran Zhang, Hao-Bo Jiang, and Hong-Bo Sun. A sers-active microfluidic device with tunable surface plasmon resonances. Electrophoresis, 32(23):3378-3384, 2011.

[43] Xiaoli Wang, Philippe Gogol, Edmond Cambril, and Bruno Palpant. Near-and far-field effects on the plasmon coupling in gold nanoparticle arrays. J. Phys. Chem. C, 116(46):24741-24747, 2012.

[44] Semion K Saikin, Yizhuo Chu, Dmitrij Rappoport, Kenneth B Crozier, and Alan Aspuru-Guzik. Separation of electromagnetic and chemical contributions to surface-enhanced Raman spectra on nanoengineered plasmonic substrates. J. Phys. Chem. Lett., 1(18):2740-2746, 2010.

[45] H. Raether. Surface-Plasmons on Smooth and Rough Surfaces and on Gratings. Springer Tr Mod Phys, 1988.

[46] William L Barnes, TW Preist, SC Kitson, and JR Sambles. Physical origin of photonic energy gaps in the propagation of surface plasmons on gratings. Phys. Rev. B, 54(9):6227, 1996.

[47] Sang Woo Han, Seung Joon Lee, and Kwan Kim. Self-assembled monolayers of aromatic thiol and selenol on silver: Comparative study of adsorptivity and stability. Langmuir, 17(22):6981-6987, 2001.

[48] Jean-Paul Hugonin, Mondher Besbes, and Philippe Lalanne. Hybridization of electromagnetic numerical methods through the g-matrix algorithm. Opt. Lett., 33(14):1590-1592, 2008.

[49] Mitradeep Sarkar, Mondher Besbes, Julien Moreau, Jean-Francois Bryche, Aurore Olivéro, Gregory Barbillon, Anne-Lise Coutrot, Bernard Bartenlian, and Michael Canva. Hybrid plasmonic mode by resonant coupling of localized plasmons to propagating plasmons in a kretschmann configuration. ACS Photonics, 2(2):237-245, 2015.

[50] Katrin Kneipp, Martin Moskovits, and Harald Kneipp. Surface-enhanced Raman scattering: physics and applications, volume 103. Springer Science \& Business Media, 2006.

[51] Eric C Le Ru and Pablo G Etchegoin. Quantifying sers enhancements. MRS Bull., 38(08):631-

640,2013 